\title{
THE FINITE FIBRE PROBLEM AND AN INDEX FORMULA FOR ELEMENTARY OPERATORS
}

\author{
JÖRG ESCHMEIER AND MIHAI PUTINAR
}

(Communicated by Palle E. T. Jorgensen)

\begin{abstract}
Let $J \subset L(K, H)$ be an operator ideal between Hilbert spaces, and let $S \in L(H)^{n}, T \in L(K)^{n}$ be commuting tuples of continuous linear operators. It is shown that the elementary operator $R: J \rightarrow J, A \rightarrow \sum_{i=1}^{n} S_{i} A T_{i}$ determined by $S$ and $T$ satisfies the finite fibre property. As a consequence it follows that an index formula proved by $L$. Fialkow for elementary operators under the additional assumption of the finite fibre property holds true in general.
\end{abstract}

Let $T \in L(X)^{n}$ be a commuting tuple of continuous linear operators on a complex Banach space $X$. We denote by $\sigma(T)$ and $\sigma_{e}(T)$ the joint spectrum and the essential joint spectrum of $T$, respectively, in the sense of J. L. Taylor (see the monograph [11] for details). If $J \subset L(K, H)$ is an operator ideal between Hilbert spaces and $S \in L(H)^{n}, T \in L(K)^{m}$ are commuting tuples of continuous linear operators, then the spectrum and essential spectrum of the multiplication tuple $M=\left(L_{S}, R_{T}\right) \in L(J)^{n+m}$, where $L_{S}=\left(L_{S_{1}}, \ldots, L_{S_{n}}\right)$ and $R_{T}=\left(R_{T_{1}}, \ldots, R_{T_{m}}\right)$ consist of the left and right multiplication operators $L_{S_{i}}: J \rightarrow J, A \rightarrow S_{i} A$ and $R_{T_{i}}: J \rightarrow J, A \rightarrow A T_{i}$, respectively, are given by (see [2], [5])

$$
\begin{aligned}
\sigma(M) & =\sigma(S) \times \sigma(T), \\
\sigma_{e}(M) & =\sigma_{e}(S) \times \sigma(T) \cup \sigma(S) \times \sigma_{e}(T) .
\end{aligned}
$$

If $n=m$ and $p$ is the polynomial in $2 n$ variables defined by $p(z, w)=$ $\sum_{i=1}^{n} z_{i} w_{i}, z, w \in \mathbb{C}^{n}$, then the resulting operator

$$
R=p(M)=\sum_{i=1}^{n} L_{S_{i}} R_{T_{i}} \in L(J)
$$

is usually called the elementary operator determined by $S$ and $T$. In [7] L. Fialkow showed how to compute the index of $\lambda-R$ for each point $\lambda$ in

Received by the editors September 28, 1992 and, in revised form, May 24, 1993.

1991 Mathematics Subject Classification. Primary 47A13; Secondary 47B47, 47A53.

This note was completed while the second author received a research fellowship from the Alexander von Humboldt Foundation, Germany. 
$\sigma(R) \backslash \sigma_{e}(R)$ satisfying the following finite fibre property:

$$
\begin{aligned}
& M_{\lambda}=\{(\alpha, \beta) \in \sigma(S) \times \sigma(T) ; p(\alpha, \beta)=\lambda\} \text { is finite, and for } \\
& \text { each pair }(\alpha, \beta) \in M_{\lambda} \text { the first component } \alpha \text { is isolated in } \\
& \sigma(S) \text { or the second component } \beta \text { is isolated in } \sigma(T) .
\end{aligned}
$$

More precisely, let $\pi, \rho: \mathbb{C}^{2 n} \rightarrow \mathbb{C}^{n}$ be the projection of $\mathbb{C}^{2 n}$ onto its first, respectively last, $n$ variables. If $\Gamma$ denotes the set of all points $\alpha$ in $\pi\left(M_{\lambda}\right)$ which are isolated in $\sigma(S)$ and $\Delta$ is the set of all points $\beta$ in $\rho\left(M_{\lambda}\right)$ for which there is a nonisolated point $\alpha$ in $\sigma(S)$ with $(\alpha, \beta) \in M_{\lambda}$, then

$$
\begin{aligned}
\operatorname{ind}(\lambda-R)= & \sum_{\beta \in \Delta} \operatorname{dim} Y(\beta) \operatorname{ind}(\lambda-p(S, \beta)) \\
& -\sum_{\alpha \in \Gamma} \operatorname{dim} X(\alpha) \operatorname{ind}(\lambda-p(\alpha, T)) .
\end{aligned}
$$

Here $Y(\beta)$ and $X(\alpha)$ denote the spectral subspaces of $T$ and $S$ corresponding to the isolated nonessential spectral points $\alpha$ and $\beta$.

In [4] it is shown that the above formula remains true, if $m, n \geq 1$ are arbitrary, $p$ is replaced by an arbitrary analytic function $f \in \mathscr{O}(\sigma(\bar{M}))$ and $\lambda \in \sigma(f(M)) \backslash \sigma_{e}(f(M))$ is a point satisfying the above finite fibre property. Moreover, the same index formula was shown to be true for operator ideals between Banach spaces and for tensor product systems instead of multiplication tuples. In [3] Curto and Fialkow proved that the finite fibre property holds, if $p$ is the above polynomial and $S=\left(u_{1}(A), \ldots, u_{n}(A)\right), T=$ $\left(v_{1}(B), \ldots, v_{n}(B)\right)$, where $A, B \in L(H)$ are completely nonunitary contractions and $u_{1}, \ldots, u_{n}, v_{1}, \ldots, v_{n}$ are functions in $H^{\infty}(T)$ that are continuously extendable to $\sigma(A) \cap T$, respectively $\sigma(B) \cap T$.

In the following we shall show that the finite fibre property holds in full generality without any additional assumptions on $S$ or $T$. This solves Problem 5.5 formulated by $\mathbf{R}$. Curto in [1] in the positive sense. We first prove a result which implies the finiteness of $M_{\lambda}$.

Theorem. Let $T \in L(X)^{n}$ be a commuting tuple of continuous linear operators on a complex Banach space $X$, and let $f \in \mathscr{O}(U)^{m}$ be an m-tuple of analytic functions defined on an open neighbourhood $U$ of $\sigma(T)$. Then for each point $\lambda \in \sigma(f(T)) \backslash \sigma_{e}(f(T))$ the fibre $f^{-1}(\lambda) \cap \sigma(T)$ is finite.

Proof. The Koszul complex regarded as an analytically parametrized complex of Banach spaces induces a sequence $K^{\cdot}\left(z-T, \mathscr{O}^{X}\right)$ of Banach free $\mathscr{O}_{\mathbb{C}^{n} \text {-sheaves. }}$ The cohomology sheaves $\mathscr{H}^{\nu}=\mathscr{H}^{\nu}\left(z-T, \mathscr{O}^{X}\right), \nu=0, \ldots, n$, are coherent analytic sheaves on the essential resolvent set $\rho_{e}(T)=\mathbb{C}^{n} \backslash \sigma_{e}(T)$ of $T$ (see [9, 10]). Since the Fredholm spectrum of $T$, i.e., the set $\sigma_{F}(T)=\sigma(T) \backslash \sigma_{e}(T)$, can be written as

$$
\sigma_{F}(T)=\bigcup_{\nu=0}^{n} \operatorname{supp}\left(\mathscr{H}^{\nu} \mid \rho_{e}(T)\right),
$$

it follows ( $\S \mathrm{IV} .1$ in [8]) that $\sigma_{F}(T)$ is an analytic subset of $\rho_{e}(T)$.

Since for $f \in \mathscr{O}(U)^{m}$ the essential spectral mapping theorem $\sigma_{e}(f(T))=$ $f\left(\sigma_{e}(T)\right)$ holds (see [6]), for each $w \in \sigma_{F}(f(T))$ the fibre $f^{-1}(w) \cap \sigma(T)$ is a compact analytic and hence finite subset of $\mathbb{C}^{n}$. 
This observation together with elementary dimension theory for complex spaces (e.g., Chapter 5 in [8]) allow us to prove the finite fibre property in full generality. In the following let us assume that $S \in L(X)^{n}, T \in L(Y)^{m}$, $M \in L(Z)^{n+m}$ are commuting tuples on Banach spaces with

$$
\begin{aligned}
\sigma(S) & \times \sigma(T) \subset \sigma(M), \\
\sigma_{e}(S) & \times \sigma(T) \cup \sigma(S) \times \sigma_{e}(T) \subset \sigma_{e}(M) .
\end{aligned}
$$

Corollary. If $f \in \mathscr{O}(\sigma(M))$ and $\lambda \in \sigma_{F}(f(M))$, then the fibre

$$
M_{\lambda}=\{(\alpha, \beta) \in \sigma(S) \times \sigma(T) ; f(\alpha, \beta)=\lambda\}
$$

is finite and for any pair $(\alpha, \beta) \in M_{\lambda}$ the first component $\alpha$ is isolated in $\sigma(S)$ or the second component $\beta$ is isolated in $\sigma(T)$.

Proof. The finiteness of $M_{\lambda}$ follows from the last theorem. Assume that $(\alpha, \beta)$ $\in M_{\lambda}$ is a point such that neither $\alpha$ is isolated in $\sigma(S)$ nor $\beta$ is isolated in $\sigma(T)$. Then $\alpha$ and $\beta$ are nonisolated points of the closed complex subspaces $\sigma_{F}(S) \subset \rho_{e}(S)$, respectively $\sigma_{F}(T) \subset \rho_{e}(T)$ (see [8, Proposition 4.3]). We regard the analytic set $\sigma_{F}(S) \times \sigma_{F}(T) \subset \rho_{e}(S) \times \rho_{e}(T)$ as a closed complex subspace of $\rho_{e}(S) \times \rho_{e}(T)$ and denote by $g$ the analytic function

$$
\sigma_{F}(S) \times \sigma_{F}(T) \rightarrow \mathbb{C}, \quad(z, w) \rightarrow f(z, w)-\lambda,
$$

induced by $f-\lambda$. Note that, since all occurring complex spaces are reduced, we need not distinguish between holomorphic maps with values in $\mathbb{C}$ and their attached scalar-valued functions [8, Chapter 4, §3]. By the definition of the analytic dimension of a complex space the zero set $N(g)$ of $g$ satisfies the estimate [8, Chapter $5, \S 2]$

$$
\operatorname{dim}_{(\alpha, \beta)} N(g) \geq \operatorname{dim}\left(\sigma_{F}(S) \times \sigma_{F}(T)\right)-1 .
$$

By the product formula for the analytic dimension [8, Chapter $5 \S 3$ ]

$$
\operatorname{dim}_{(\alpha, \beta)}\left(\sigma_{F}(S) \times \sigma_{F}(T)\right)=\operatorname{dim}_{\alpha} \sigma_{F}(S)+\operatorname{dim}_{\beta} \sigma_{F}(T),
$$

and by our assumption that $\alpha$ and $\beta$ are nonisolated it follows that the righthand side is larger or equal to 2 . Therefore, $\operatorname{dim}_{(\alpha, \beta)} N(g) \geq 1$ and hence $(\alpha, \beta)$ is not isolated in $N(g)$. In particular, the function $g$ would have infinitely many zeros, which contradicts the finiteness of $M_{\lambda}$.

As a consequence of the last result the index formulas cited above (Theorem 1.1 in [7] and Theorem D in [4]) are valid without any extra assumption on the fibre sets $M_{\lambda}$. Moreover, the last proof shows that in the setting of the corollary

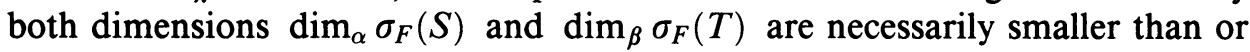
equal to one.

\section{REFERENCES}

1. R. Curto, Spectral theory of elementary operators, Elementary Operators \& Applications, Proceedings of the International Workshop (M. Mathieu, ed.), World Scientific, Singapore, 1992, pp. 3-52.

2. R. Curto and L. Fialkow, The spectral picture of $\left(L_{A}, R_{B}\right)$, J. Funct. Anal. 71 (1987), 371-392.

3. _ Elementary operators with $H^{\infty}$-symbols, Integral Equations Operator Theory 10 (1987), 707-720. 
4. J. Eschmeier, Analytic index formulas for tensor product systems, Proc. Roy. Irish Acad. Sect. A 87 (1987), 121-135.

5. __ Tensor products and elementary operators, J. Reine Angew. Math. 390 (1988), 47-66.

6. A. S. Fainshtein, Joint essential spectrum of a family of linear operators, Funktsional Anal. i Prilozhen. 14 (1980), 83-84; English transl., Functional Anal. Appl. 14 (1980), 152-153.

7. L. Fialkow, The index of an elementary operator, Indiana Univ. Math. J. 35 (1986), 73-102.

8. H. Grauert and R. Remmert, Coherent analytic sheaves, Springer-Verlag, Berlin, 1984.

9. R. N. Levy, Algebraic and topological K-functors of commuting $n$-tuple of operators, J. Operator Theory 21 (1989), 219-253.

10. M. Putinar, Base change and the Fredholm index, Integral Equations Operator Theory 8 (1985), 674-692.

11. F.-H. Vasilescu, Analytic functional calculus and spectral decompositions, Reidel, Dordrecht, 1982.

Mathematisches Institut, Universität Münster, D-48149 MÜNSTeR, GeRmaNy

E-mail address, J. Eschmeier: eschmei $0_{\text {math }}$ uni-muenster.de

Department of Mathematics, University of California, Riverside, California 92521

E-mail address, M. Putinar: mputinar@ucrmath.ucr.edu 